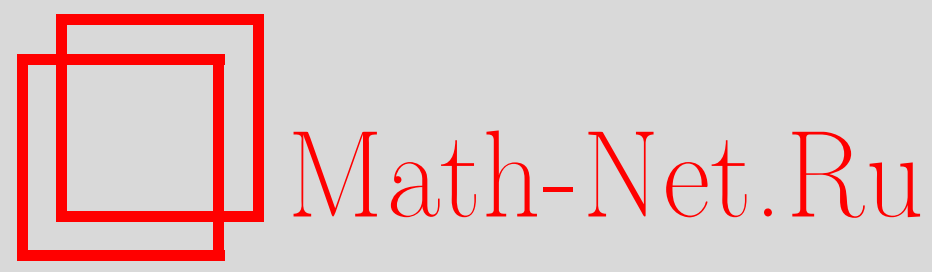

Е. Б. Титова, В. Н. Шевченко, О минорах матрицы ограничений многоиндексных транспортных задач, Дискрет. матем., 2012, том 24, выпуск 4, 147-157

DOI: https://doi.org/10.4213/dm1217

Использование Общероссийского математического портала Math-Net.Ru подразумевает, что вы прочитали и согласны с пользовательским соглашением http://www . mathnet.ru/rus/agreement

Параметры загрузки:

IP : 54.84 .234 .179

26 апреля 2023 г., 14:46:35 
УДК 519.8

\title{
О минорах матрицы ограничений многоиндексных транспортных задач
}

\author{
() 2012 г. Е. Б. Титова, В.Н.Шевченко
}

Для матрицы $T_{s, k}(n)$ ограничений $k$-индексной $s$-арной транспортной задачи, в которой каждый индекс принимает $n$ значений, рассмотрено поведение функции $\alpha_{j}\left(T_{s, k}(n)\right)$, равной среднему значению квадрата минора $j$-го порядка матрицы $T_{s, k}(n)$. Найдены двусторонние оценки логарифма величины $\alpha_{j}\left(T_{s, k}(n)\right)$. На их основе получена классификация значений порядка $j$, при которых $\left.\alpha_{j}\left(T_{s, k}(n)\right)\right)$ стремится к бесконечности, и значений, при которых $\alpha_{j}\left(T_{s, k}(n)\right)$ стремится к нулю. Дана оценка максимальной величины миноров матрицы $T_{s, k}(n)$. Найдена асимптотика функции $\alpha_{\mathscr{A}}\left(T_{s, k}(n)\right)$, равной среднему значению квадрата минора рангового порядка базисной системы строк матрицы $T_{s, k}(n)$. Большинство этих результатов были анонсированы ранее без доказательств.

Работа поддержана Российским фондом фундаментальных исследований, проект 09-01-00545-a.

1. Обозначим через $\mathbf{Z}$ кольцо целых чисел, через $\mathbf{Q}$ - поле рациональных чисел, через $\mathbf{F}^{p \times q}-$ множество $(p \times q)$-матриц с элементами из множества $F$, через $\mathbf{1}^{p \times q}$ и $\mathbf{0}^{p \times q}$ - $(p \times q)$-матрицы, у которых все элементы равны 1 и 0 соответственно, через $E_{n}-$ единичную матрицу $n$-го порядка, через $e_{j}(n)-j$-й столбец единичной матрицы $E_{n}$.

Если $I \subseteq\{1, \ldots, p\}$ и $J=\{1, \ldots, q\}$, то через $A(I, J)$ обозначим подматрицу матрицы $A$ со строками из множества $I$ и столбцами из множества $J$, через $M_{k v}(A)-$ множество миноров $k$-го порядка матрицы $A$, равных $v$ по абсолютной величине, то есть

$$
M_{k v}(A)=\{(I, J)|| I|=| J|=k,| \operatorname{det} A(I, J) \mid=v\},
$$

и положим

$$
\begin{aligned}
x_{k v} & =\left|M_{k v}(A)\right|, \\
\delta_{k}(A) & =\max \left\{v \mid x_{k v} \neq 0\right\}, \\
\delta(A) & =\max \left\{\delta_{k}(A), k=1, \ldots, r\right\},
\end{aligned}
$$

где $r$ - ранг матрицы $A$. Обозначим матрицу, транспонированную к $A$, через $A^{\top}$, и положим

$$
\operatorname{det}\left(\lambda E_{q}-A^{\top} A\right)=\sum_{k=0}^{q}(-1)^{k} s_{k}(A) \lambda^{q-k}=\lambda^{q-r} \prod_{i=1}^{l}\left(\lambda-\lambda_{i}\right)^{m_{i}},
$$

где $\lambda_{i}, i=1, \ldots, l,-$ различные положительные собственные числа с кратностями $m_{i}$. 
Хорошо известно (см., например, [1]), что ненулевые собственные числа матриц $A A^{\top}$ и $A^{\top} A$ совпадают, причем, если $f-$ ненулевой собственный вектор для $A^{\top} A$, соответствующий ненулевому собственному значению $\lambda$, то $A f$ - собственный вектор для $A A^{\top}$ с тем же собственным значением.

Из формулы Бине-Коши (см., например, $[1,2])$ следует, что

$$
s_{k}(A)=\sum_{|I|=k} \sum_{|J|=k} \operatorname{det}^{2} A(I, J) .
$$

В частности, если $r=p$, то

$$
\operatorname{det} A^{\top} A=\sum_{\nu=1}^{\delta_{r}(A)} v^{2} x_{k v}(A),
$$

что позволяет оценить величину

$$
\alpha_{r}(A)=s_{r}(A)\left(\begin{array}{l}
q \\
r
\end{array}\right)^{-1},
$$

равную среднему значению квадрата базисного минора матрицы $A$.

Если матрица $A$ не является унимодулярной, то для оценки роста знаменателя необходимо знать поведение величины $\delta(A)$. Для полиэдра

$$
\mathbf{P}(A, b)=\{A x=b, x \geqslant 0\},
$$

достаточно рассмотреть лишь базисные миноры матрицы $A$. Если же многогранник задан в форме неравенств, то необходима информация о минорах произвольного порядка.

В теории линейных неравенств [3] известен алгоритм построения системы линейных относительно параметра $b$ неравенств, дающий критерий непустоты полиэдра $\mathbf{P}(A, b)$. Так как коэффициенты этих неравенств связаны с минорами $(r-1)$-го порядка матрицы $A$, понятен интерес к оценке величины $\delta_{r-1}(A)$.

Множество

$$
R_{A}=\left\{x \in \mathbf{Q}^{q} \mid A^{\top} A x=0\right\}=\left\{x \in \mathbf{Q}^{q} \mid A x=0\right\}
$$

назовем правым нуль-пространством матрицы $A$, а множество

$$
R_{A}^{\mathbf{Z}}=R_{A} \cap \mathbf{Z}^{N}
$$

- правым модулем матрицы $A$.

Будем считать, что базис пространства $R_{A}$ задан матрицей $B \in \mathbf{Z}^{q \times(q-r)}$. Ясно, что множество целочисленных линейных комбинаций столбцов матрицы $B$ содержится в $R_{A}^{Z}$, но, вообще говоря, с ним не совпадает. Это выполняется тогда и только тогда, когда матрица $B$ унимодулярна (то есть, наибольший общий делитель базисных миноров равен единице); в этом случае назовем ее базисом модуля $R_{A}^{\mathbf{Z}}$.

Известно, что если матрица $A$ содержит базисную систему строк $A^{\mathscr{A}}$, имеющую минор порядка $r$, равный 1, то (см., например, [4])

$$
\left|\operatorname{det} A^{\mathscr{A}}(\mathscr{\mathscr { F }})\right|=\left|\operatorname{det} R_{A}^{\mathbf{Z}}(\overline{\mathscr{F}})\right|,
$$

где $\overline{\mathscr{F}}-$ множество, дополнительное к $\mathscr{\mathscr { g }}$, и, следовательно, количественный анализ базисных миноров матриц $A^{\mathscr{A}}$ и $R_{A}^{\mathbf{Z}}$ равносилен. 
В настоящей статье продолжены исследования из [5] для матрицы $A=T_{s, k}(n)$ ограничений многоиндексной транспортной задачи (Т3), реализующие предложенное в [6] использование найденного там многочлена матрицы $A A^{\top}$. Если $A$ совпадает с матрицей $T_{s, k}(n)$ многоиндексной транспортной задачи, то $\operatorname{det}\left(\lambda E_{q}-A A^{\top}\right)$ совпадает (см. [6]) с многочленом

$$
\Delta(\lambda ; s, k, n)=\lambda^{\sum_{i=0}^{s-1}\left(\begin{array}{c}
k \\
i
\end{array}\right)(n-1)^{k-i}}\left(\prod_{j=s}^{k}\left(\lambda-\left(\begin{array}{l}
j \\
s
\end{array}\right) n^{s}\right)\right)^{\left(\begin{array}{c}
k \\
j
\end{array}\right)(n-1)^{k-j}} .
$$

2. Далее рассмотрим многогранники транспортных задач, задаваемые соотношением $T x=b$ (или $T x \leqslant b), x \geqslant 0$, где вектор $x$ имеет $k$-индексные компоненты $x_{J}=x_{j_{1}, \ldots, j_{k}}$, $j_{i} \in\left\{1, \ldots, n_{i}\right\}, i=1, \ldots, k$, а строки матрицы $T=T_{s, k}\left(n_{1}, \ldots, n_{k}\right)$ разбиты на ярусы $T_{I}$, где $I$ есть $s$-элементное подмножество множества $\{1, \ldots, k\}$, задающее набор индексов $j_{i}, i \in I$, по которым ведется суммирование, первые из которых, $I_{1}=\{1, \ldots, s\}$ соответствуют сумме

$$
T_{I_{1}} x=\sum_{j_{1}=1}^{n_{1}} \cdots \sum_{j_{s}=1}^{n_{S}} x_{j_{1}, \ldots, j_{k}} .
$$

В [6] ярусы $T_{I}$ выражены через кронекерово произведение матриц $\mathbf{1}^{1 \times n}$ и $E_{n}$ следующим образом:

$$
T_{I}=A_{I}\left(1, n_{1}\right) \otimes \ldots \otimes A_{I}\left(k, n_{k}\right),
$$

где $A_{I}\left(v, n_{v}\right)=\mathbf{1}^{1 \times n_{v}}$ при $v \in I$ и $A_{I}\left(v, n_{v}\right)=E_{n_{v}}$ при $v \notin I$. В частности,

$$
T_{I_{1}}=\mathbf{1}^{1 \times n_{1}} \otimes \ldots \otimes 1^{1 \times n_{s}} \otimes E_{n_{s+1}} \otimes \ldots \otimes E_{n_{k}} .
$$

Напомним, что кронекеровым произведением (определение и свойства см., например, в [2] $),(m \times n)$-матрицы $A$ и $(p \times q)$-матрицы $B$ называется $(p m \times q n)$-матрица, имеющая блочный вид

$$
A \otimes B=\left(\begin{array}{ccc}
a_{11} B & \cdots & a_{1 n} B \\
\ldots \ldots \ldots & \ldots & \ldots \ldots \\
a_{m 1} B & \cdots & a_{m n} B
\end{array}\right) .
$$

Кронекерово произведение обладает следующими свойствами:

$$
\begin{aligned}
(A \otimes B)(C \otimes D) & =A C \otimes B D, \\
(A \otimes B)^{T} & =A^{T} \otimes B^{T} .
\end{aligned}
$$

В матрице $T_{s, k}\left(n_{1}, \ldots, n_{k}\right)$ число блоков равно $\left(\begin{array}{l}k \\ s\end{array}\right)$, число столбцов равно

$$
N=\prod_{i=1}^{k} n_{i}
$$

и число строк равно

$$
M=\sigma_{k-s}\left(n_{1}, \ldots, n_{k}\right)
$$


где

$$
\sigma_{s}\left(\alpha_{1}, \ldots, \alpha_{k}\right)=\sum_{1 \leqslant i_{1}<\ldots<i_{s} \leqslant k} \alpha_{i_{1}} \alpha_{i_{2}} \ldots \alpha_{i_{s}}
$$

есть $s$-я элементарная симметрическая функция от $\alpha_{1}, \ldots, \alpha_{k}$, причем

$$
\sigma_{0}\left(\alpha_{1}, \ldots, \alpha_{k}\right)=1 \text {. }
$$

Итак, обычная транспортная задача в этих обозначениях имеет матрицу

$$
T_{1,2}\left(n_{1}, n_{2}\right)=\left[\frac{1^{1 \times n_{1}} \otimes E_{n_{2}}}{E_{n_{1}} \otimes 1^{1 \times n_{2}}}\right] .
$$

Для нее Симмонард и Хедли нашли (см., например [7]) число базисов, воспользовавшись вполне унимодулярностью матрицы $T_{1,2}\left(n_{1}, n_{2}\right)$.

При $k \geqslant 3$ в транспортном многограннике могут появиться вершины, имеющие нецелые координаты, и для оценки роста знаменателя необходимо знать поведение величины $\delta\left(T_{s, k}\right)$.

Пусть

$$
Q_{n}=\left(\begin{array}{rrrr}
1 & 1 & \ldots & 1 \\
1 & -1 & \ldots & 0 \\
\ldots & \ldots & \ldots & \ldots \\
1 & 0 & \ldots & -1
\end{array}\right)
$$

есть матрица $n$-го порядка, и $q_{j}$ есть ее $j$-й столбец. Обозначим через $q_{J}$, где $J=\left\{j_{1}, \ldots, j_{k}\right\}, J$-й столбец матрицы $Q=Q_{n_{1}} \otimes \ldots \otimes Q_{n_{k}}$. Очевидно, что

$$
q_{J}=q\left(j_{1}, n_{1}\right) \otimes \ldots \otimes q\left(j_{k}, n_{k}\right) .
$$

Далее используем сокращенное обозначение

$$
T_{s, k}\left(n_{1}, \ldots, n_{k}\right)=T_{s, k},
$$

если это не будет приводить к недоразумению.

Предложение 1 ([6]). Столбизь матрицы $Q=Q_{n_{1}} \otimes \ldots \otimes Q_{n_{k}}$ составляют базис из собственных векторов матрицы $T_{s, k}^{\top} T_{s, k}$.

Разумеется, собственные векторы можно выбирать и по-другому, однако предложенный способ дает целочисленную и симметричную матрицу $Q_{n}$. Для матриц $T_{s, k} T_{s, k}^{\top}$ непосредственно воспользоваться кронекеровым произведением удается лишь в случае $s=k-1$.

Предложение 2 ([8]). Столбиь матрицыь $Q=Q_{k} \otimes Q_{n}$ составляют базис из собственных векторов матрицы $T_{k-1, k} T_{k-1, k}^{\top}$.

Матрицу, получающуюся из $Q_{n}$ заменой ее первого столбца на $e_{1}$, обозначим через $\bar{Q}_{n}$, и положим

$$
\bar{Q}=\bar{Q}_{n_{1}} \otimes \ldots \otimes \bar{Q}_{n_{k}}
$$

Удалим из $\bar{Q}$ все те ее столбцы $\bar{q}_{j}$, для которых $J$ не удовлетворяет условию, что для всех $\tau$ найдется $\mu \in I_{\tau}, j_{\mu} \neq 1$, и оставшуюся после этого матрицу обозначим через $R_{s, k}$. Эта матрица является базисом модуля $R_{T}^{\mathrm{Z}}$ (см. [9]). 
Известно, что матрица $T_{s, k}$ содержит базисную систему строк $T_{s, k}^{A},|\mathscr{A}|=r_{s, k}$, имеющую минор порядка $r_{s, k}$, равный 1. Очевидно, что

$$
T_{s, k} R_{s, k}=0 \Longleftrightarrow T_{s, k}^{\mathscr{A}} R_{s, k}=0 .
$$

Известно [4], что

$$
\left|\operatorname{det}\left(R_{s, k}^{\top} R_{s, k}\right)\right|=\left|\operatorname{det}\left(T_{s, k}^{\not A} T_{s, k}^{s \top}\right)\right| .
$$

Поэтому при исследовании поведения среднего значения $\alpha_{\mathfrak{A}}\left(T_{s, k}(n)\right)$ квадрата минора матрицы $T_{s, k}^{\mathscr{A}}(n)$ мы используем значение определителя Грама матрицы $R_{s, k}$.

В настоящей работе нами получены двусторонние оценки логарифма среднего значения $\alpha_{j}\left(T_{s, k}(n)\right)$ квадрата минора матрицы $T_{s, k}(n)$ произвольного порядка $j$ и доказано, что $\alpha_{j}\left(T_{s, k}(n)\right) \rightarrow \infty$ с экспоненциальной скоростью при $j \sim \theta r, e /\left(\begin{array}{l}k \\ s\end{array}\right)<\theta \leqslant 1$, где $e$ - основание натуральных логарифмов, и $\alpha_{j}\left(T_{s, k}(n)\right) \rightarrow 0$ с экспоненциальной скоростью при $j \sim \theta r, 0 \leqslant \theta<e /\left(\begin{array}{l}k \\ s\end{array}\right)$. Вычислен определитель Грама матрицы $R_{s, k}$, найдена асимптотика величины $\alpha_{\mathscr{A}}\left(T_{s, k}(n)\right)$ и доказано, что $\alpha_{\mathscr{A}}\left(T_{s, k}(n)\right) \rightarrow \infty$ с экспоненциальной скоростью. Результаты были анонсированы в [10-16].

3. Рассмотрим поведение среднего значения квадрата миноров матрицы $T_{s, k}(n)$ произвольного порядка $j$. В [16] были доказаны следующие утверждения.

Лемма 1. Для любого $j=1, \ldots, r$ справедливы неравенства

$$
j\left(\ln \frac{j}{e n^{k-s}}-\frac{f_{n}}{j}\right) \leqslant \ln \alpha_{j}\left(T_{s, k}(n)\right) \leqslant j\left(\ln \frac{j}{e n^{k-s}}+\gamma_{n}+\frac{\ln \sqrt{2 \pi e j}}{j}\right),
$$

где

$$
f_{n}=s\left(\begin{array}{c}
k \\
s+1
\end{array}\right)(n-1)^{k-s-1}\left(1+\varepsilon_{n}\right)\left(\ln \left(\begin{array}{l}
k \\
s
\end{array}\right)+(k-s) \ln n\right)=o(r) .
$$

Теорема 1. При фиксированных $k \geqslant 3,1 \leqslant s \leqslant k-1$, для средней величиньл $\alpha_{j}\left(T_{s, k}(n)\right)$ квадрата минора $j$-го порядка матрищы $T_{s, k}(n)$ справедливы следующие утверждения:

(1) $\alpha_{j}\left(T_{s, k}(n)\right) \rightarrow 0$ при $j=o(r)$;

(2) $\alpha_{j}\left(T_{s, k}(n)\right) \rightarrow 0$ при $j \sim \theta r$, где $0<\theta<e\left(\begin{array}{l}k \\ s\end{array}\right)^{-1}$;

(3) $\alpha_{j}\left(T_{s, k}(n)\right) \rightarrow \infty$ при j $\sim \theta r$, где $e\left(\begin{array}{l}k \\ s\end{array}\right)^{-1}<\theta \leqslant 1$.

Следствие 1. При $\left(\begin{array}{l}k \\ s\end{array}\right) \geqslant e^{2}$, среднее значение модуля $\varphi_{r}\left(T_{s, k}(n)\right)$ минора рангового порядка матрицы $T_{s, k}(n)$ стремится к бесконечности с экспоненџиальной скоростью.

Для доказательства достаточно воспользоваться очевидным неравенством

$$
s_{r}\left(T_{s, k}(n)\right) \leqslant \delta_{r}\left(T_{s, k}(n)\right) \varphi_{r}\left(T_{s, k}(n)\right) .
$$

Аналогично доказывается следствие 2.

Следствие 2. При $\left(\begin{array}{l}k \\ s\end{array}\right) \geqslant e^{2}$, среднее значение модуля $\varphi_{r-1}\left(T_{s, k}(n)\right)$ минора предрангового порядка матрищы $T_{s, k}(n)$ стремится к бесконечности с экспоненциильной скоростью.

Следует отметить, что в случае, когда значение $k-s$ невелико, удается получить асимптотику самой величины $\alpha_{j}\left(T_{s, k}(n)\right)$ (см. [10]). При $k=3$, как при $s=1$, так и при $s=2$, в [17] установлен экспоненциальный рост величины $\delta_{r}\left(T_{s, 3}(n)\right)$ для ранговых миноров. 
4. Вычислим определитель Грама матрицы $R_{s, k}$. Если

$$
Q_{n}^{*}=\left[\begin{array}{rrr}
1 & \ldots & 1 \\
-1 & \ldots & 0 \\
\ldots & \ldots & \ldots \\
0 & \ldots & -1
\end{array}\right]=\left[\frac{1^{1 \times(n-1)}}{-E_{n-1}}\right]
$$

есть матрица порядка $n \times(n-1)$, то заметим, что

$$
Q_{n}=\left[1^{n \times 1} \mid Q_{n}^{*}\right], \quad \bar{Q}_{n}=\left[e_{1}(n) \mid Q_{n}^{*}\right]
$$

Лемма 2 ([15]). Базис правого модуля матрицы $T_{1, k}\left(n_{1}, \ldots, n_{k}\right)$ планарной транспортной задачи составляют столбцы матрищьы $R_{1, k}\left(n_{1}, \ldots, n_{k}\right)=Q_{n_{1}}^{*} \otimes \ldots \otimes Q_{n_{k}}^{*}$. Характеристический многочлен есть

$$
\begin{aligned}
& \Delta\left(R_{1, k}^{\top}\left(n_{1}, \ldots, n_{k}\right) R_{1, k}\left(n_{1}, \ldots, n_{k}\right)\right)=\left(\lambda-n_{1} \cdots n_{k}\right) \\
& \times \prod_{i=1}^{k}\left(\lambda-n_{1} \cdots n_{i-1} n_{i+1} \cdots n_{k}\right)^{n_{i}-2} \cdots \prod_{i=1}^{k}\left(\lambda-n_{i}\right) \prod_{\substack{j \neq 1 \\
j \neq i}}^{\left(n_{j}-2\right)}(\lambda-1)^{\prod_{i=1}^{k}\left(n_{i}-2\right)} .
\end{aligned}
$$

Следствие 3. При $n_{1}=\ldots=n_{k}=n$,

$$
\operatorname{det}\left(R_{1, k}^{\top} R_{1, k}\right)=n^{k(n-1)^{k-1}} .
$$

Нетрудно проверить справедливость следующего утверждения.

Лемма 3. Для произвольных $k$ u $1 \leqslant s \leqslant k-1$, матрииу правого модуля можно представить в виде двух столбиовых блоков:

$$
R_{s, k}\left(n_{1}, \ldots, n_{k}\right)=\left[Q_{n_{1}}^{*} \otimes R_{s, k-1}\left(n_{2}, \ldots, n_{k}\right) \mid e_{1}\left(n_{1}\right) \otimes R_{s-1, k-1}\left(n_{2}, \ldots, n_{k}\right)\right]
$$

2de

$$
\begin{aligned}
R_{1, k}\left(n_{1}, \ldots, n_{k}\right) & =Q_{n_{1}}^{*} \otimes \ldots \otimes Q_{n_{k}}^{*}, \\
R\left(k, k, n_{1}, \ldots, n_{k}\right) & =Q_{n_{1} \cdots n_{k}}^{*} .
\end{aligned}
$$

Далее везде будем считать, что $n_{1}=\ldots=n_{k}=n$.

Теорема 2. Справедлива рекурсивная формула

$$
\operatorname{det}\left(R_{s, k}^{\top} R_{s, k}\right)=n^{\left(\begin{array}{c}
k-1 \\
s-1
\end{array}\right)(n-1)^{k-s}} \operatorname{det}^{n-1}\left(R_{s, k-1}^{\top} R_{s, k-1}\right) \operatorname{det}\left(R_{s-1, k-1}^{\top} R_{s-1, k-1}\right) .
$$

Доказательство. Рассмотрим произведение матриц

$$
\begin{aligned}
R_{s, k}^{\top} R_{s, k} & =\left[\frac{Q_{n}^{* \top} \otimes R_{s, k-1}^{\top}}{e_{1}(n)^{\top} \otimes R_{s-1, k-1}^{\top}}\right]\left[Q_{n}^{*} \otimes R_{s, k-1} \mid e_{1}(n) \otimes R_{s-1, k-1}\right] \\
& =\left[\begin{array}{c|c}
\left(Q_{n}^{* \top} Q_{n}^{*}\right) \otimes\left(R_{s, k-1}^{\top} R_{s, k-1}\right) & \left(Q_{n}^{* \top} e_{1}(n)\right) \otimes\left(R_{s, k-1}^{\top} R_{s-1, k-1}\right) \\
\hline\left(e_{1}(n)^{\top} Q_{n}^{*}\right) \otimes\left(R_{s-1, k-1}^{\top} R_{s, k-1}\right) & \left(e_{1}(n)^{\top} e_{1}(n)\right) \otimes\left(R_{s-1, k-1}^{\top} R_{s-1, k-1}\right)
\end{array}\right] .
\end{aligned}
$$


Так как

$$
\begin{aligned}
Q_{n}^{* \top} e_{1}(n) & =\mathbf{1}^{(n-1) \times 1}, \\
e_{1}(n)^{\top} Q_{n}^{*} & =\mathbf{1}^{1 \times(n-1)}, \\
e_{1}(n)^{\top} e_{1}(n) & =1,
\end{aligned}
$$

получаем, что

$$
R_{s, k}^{\top} R_{s, k}=\left[\begin{array}{c|l}
\left(Q_{n}^{* \top} Q_{n}^{*}\right) \otimes\left(R_{s, k-1}^{\top} R_{s, k-1}\right) & \mathbf{1}^{(n-1) \times 1} \otimes\left(R_{s, k-1}^{\top} R_{s-1, k-1}\right) \\
\hline \mathbf{1}^{1 \times(n-1)} \otimes\left(R_{s-1, k-1}^{\top} R_{s, k-1}\right) & R_{s-1, k-1}^{\top} R_{s-1, k-1}
\end{array}\right],
$$

где

$$
\begin{aligned}
Q_{n}^{* \top} Q_{n}^{*} & =\left[\mathbf{1}^{(n-1) \times 1} \mid-E_{n-1}\right]\left[\frac{\mathbf{1}^{1 \times(n-1)}}{-E_{n-1}}\right] \\
& =\mathbf{1}^{(n-1) \times(n-1)}+E_{n-1} .
\end{aligned}
$$

Легко видеть, что собственными числами матрицы $Q_{n}^{* \top} Q_{n}^{*}$ являются $n$ кратности 1 и 1 кратности $n-2$, а матрица

$$
Q_{n-1}=\left[1^{(n-1) \times 1} \mid Q_{n-1}^{*}\right]
$$

является для нее базисом из собственных векторов, причем столбец $\mathbf{1}^{(n-1) \times 1}$ соответствует собственному числу $n$. Отсюда следует, что

$$
\left(Q_{n}^{* \top} Q_{n}^{*}\right) Q_{n-1}=Q_{n-1} D_{n-1},
$$

где

$$
D_{n-1}=\operatorname{diag}(n, 1, \ldots, 1)_{n-1} .
$$

Рассмотрим трансформирующую матрицу

$$
P=P_{r_{s, k}}=\left[\begin{array}{c|c}
Q_{n-1} \otimes E_{r g_{s, k-1}} & \mathbf{0} \\
\hline \mathbf{0} & E_{r g_{s-1, k-1}}
\end{array}\right] .
$$

Вычислим матрицу $P^{-1}\left(R_{s, k}^{\top} R_{s, k}\right) P$, подобную матрице $R_{s, k}^{\top} R_{s, k}$ :

$$
\begin{aligned}
& P^{-1}\left(R_{s, k}^{\top} R_{s, k}\right) P \\
& =\left[\begin{array}{c|c}
Q_{n-1}^{-1} \otimes E_{r g_{s, k-1}} & \mathbf{0} \\
\hline \mathbf{0} & E_{r g_{s-1, k-1}}
\end{array}\right] \\
& \times\left[\begin{array}{c|l}
\left(Q_{n}^{* \top} Q_{n}^{*}\right) \otimes\left(R_{s, k-1}^{\top} R_{s, k-1}\right) & \mathbf{1}^{(n-1) \times 1} \otimes\left(R_{s, k-1}^{\top} R_{s-1, k-1}\right) \\
\hline \mathbf{1}^{1 \times(n-1)} \otimes\left(R_{s-1, k-1}^{\top} R_{s, k-1}\right) & R_{s-1, k-1}^{\top} R_{s-1, k-1}
\end{array}\right] \\
& \times\left[\begin{array}{c|c}
Q_{n-1} \otimes E_{r g_{s, k-1}} & \mathbf{0} \\
\hline \mathbf{0} & E_{r g_{s-1, k-1}}
\end{array}\right] \\
& =\left[\begin{array}{c|c}
Q_{n-1}^{-1} \otimes E_{r g_{s, k-1}} & \mathbf{0} \\
\hline \mathbf{0} & E_{r g_{s-1, k-1}}
\end{array}\right] \\
& \times\left[\begin{array}{c|l}
\left(Q_{n-1} D_{n-1}\right) \otimes\left(R_{s, k-1}^{\top} R_{s, k-1}\right) & \mathbf{1}^{(n-1) \times 1} \otimes\left(R_{s, k-1}^{\top} R_{s-1, k-1}\right) \\
\hline\left(\mathbf{1}^{1 \times(n-1)} Q_{n-1}\right) \otimes\left(R_{s-1, k-1}^{\top} R_{s, k-1}\right) & R_{s-1, k-1}^{\top} R_{s-1, k-1}
\end{array}\right] \\
& =\left[\begin{array}{r|l}
D_{n-1} \otimes\left(R_{s, k-1}^{\top} R_{s, k-1}\right) & \left(Q_{n-1}^{-1} \mathbf{1}^{(n-1) \times 1}\right) \otimes\left(R_{s, k-1}^{\top} R_{s-1, k-1}\right) \\
\hline\left(\mathbf{1}^{1 \times(n-1)} Q_{n-1}\right) \otimes\left(R_{s-1, k-1}^{\top} R_{s, k-1}\right) & R_{s-1, k-1}^{\top} R_{s-1, k-1}
\end{array}\right] .
\end{aligned}
$$


Нетрудно видеть, что

$$
\begin{aligned}
Q_{n-1}^{-1} & =\frac{1}{n-1}\left[\begin{array}{cccc}
1 & 1 & \ldots & 1 \\
1 & 2-n & \ldots & 1 \\
\ldots & \ldots \ldots & \ldots & \ldots \ldots \\
1 & 1 & \ldots & 2-n
\end{array}\right], \\
\mathbf{1}^{1 \times(n-1)} Q_{n-1} & =(n-1) e_{1}^{\top}(n-1), \\
Q_{n-1}^{-1} \mathbf{1}^{(n-1) \times 1} & =e_{1}(n-1),
\end{aligned}
$$

тогда

$$
\begin{aligned}
& P^{-1}\left(R_{s, k}^{\top} R_{s, k}\right) P \\
& =\left[\begin{array}{c|c}
D_{n-1} \otimes\left(R_{s, k-1}^{\top} R_{s, k-1}\right) & e_{1}(n-1) \otimes\left(R_{s, k-1}^{\top} R_{s-1, k-1}\right) \\
\hline(n-1) e_{1}^{\top}(n-1) \otimes\left(R_{s-1, k-1}^{\top} R_{s, k-1}\right) & R_{s-1, k-1}^{\top} R_{s-1, k-1}
\end{array}\right] \\
& =\left[\begin{array}{c|c|c}
n\left(R_{s, k-1}^{\top} R_{s, k-1}\right) & \mathbf{0} & R_{s, k-1}^{\top} R_{s-1, k-1} \\
\hline \mathbf{0} & E_{n-2} \otimes\left(R_{s, k-1}^{\top} R_{s, k-1}\right) & \mathbf{0} \\
\hline(n-1)\left(R_{s-1, k-1}^{\top} R_{s, k-1}\right) & \mathbf{0} & R_{s-1, k-1}^{\top} R_{s-1, k-1}
\end{array}\right] .
\end{aligned}
$$

Определители подобных матриц равны, поэтому

$$
\begin{aligned}
& \operatorname{det}\left(R_{s, k}^{\top} R_{s, k}\right)= \operatorname{det}\left(P^{-1}\left(R_{s, k}^{\top} R_{s, k}\right) P\right) \\
&= \operatorname{det}\left[E_{n-2} \otimes\left(R_{s, k-1}^{\top} R_{s, k-1}\right)\right] \\
& \quad \times \operatorname{det}\left[\begin{array}{r|r}
n\left(R_{s, k-1}^{\top} R_{s, k-1}\right) & R_{s, k-1}^{\top} R_{s-1, k-1} \\
\hline(n-1)\left(R_{s-1, k-1}^{\top} R_{s, k-1}\right) & R_{s-1, k-1}^{\top} R_{s-1, k-1}
\end{array}\right] \\
&=\operatorname{det}^{n-2}\left(R_{s, k-1}^{\top} R_{s, k-1}\right) \\
& \quad \times \operatorname{det}\left[\begin{array}{r|r}
n\left(R_{s, k-1}^{\top} R_{s, k-1}\right) & R_{s, k-1}^{\top} R_{s-1, k-1} \\
\hline(n-1)\left(R_{s-1, k-1}^{\top} R_{s, k-1}\right) & R_{s-1, k-1}^{\top} R_{s-1, k-1}
\end{array}\right] .
\end{aligned}
$$

Далее, заметим, что матрица

$$
R_{s-1, k-1}=R_{s, k-1}\left[\frac{E_{r g_{s-1, k-1}}}{\mathbf{0}_{r g_{s, k-1}-r_{s-1, k-1} \otimes r g_{s-1, k-1}}}\right]
$$

с точностью до перестановки столбцов. Отсюда видно, что каждый столбец из правого верхнего блока матрицы из правой части (7) присутствует с коэффициентом $n$ и в левом верхнем блоке этой матрицы, а каждый столбец из правого нижнего блока присутствует с коэффициентом $(n-1)$ и в левом нижнем блоке. Вычтем из каждого столбца правой полосы этой матрицы соответствующий столбец левой полосы с коэффициентом $1 / n$. 
Тогда получим, что

$$
\begin{aligned}
\operatorname{det}\left(R_{s, k}^{\top} R_{s, k}\right)= & \operatorname{det}^{n-2}\left(R_{s, k-1}^{\top} R_{s, k-1}\right) \\
& \times \operatorname{det}\left[\begin{array}{c|c}
n\left(R_{s, k-1}^{\top} R_{s, k-1}\right) & \mathbf{0} \\
\hline(n-1)\left(R_{s-1, k-1}^{\top} R_{s, k-1}\right) & \frac{1}{n}\left(R_{s-1, k-1}^{\top} R_{s-1, k-1}\right)
\end{array}\right] \\
= & \operatorname{det}^{n-2}\left(R_{s, k-1}^{\top} R_{s, k-1}\right) n^{r g_{s, k-1}} \operatorname{det}\left(R_{s, k-1}^{\top} R_{s, k-1}\right)\left(\frac{1}{n}\right)^{r g_{s-1, k-1}} \\
& \times \operatorname{det}\left(R_{s-1, k-1}^{\top} R_{s-1, k-1}\right) \\
= & n^{r g_{s, k-1}-r g_{s-1, k-1} \operatorname{det}^{n-1}\left(R_{s, k-1}^{\top} R_{s, k-1}\right) \operatorname{det}\left(R_{s-1, k-1}^{\top} R_{s-1, k-1}\right) .}
\end{aligned}
$$

Так как

$$
\begin{aligned}
r g_{s, k} & =n^{k}-r_{s, k} \\
& =n^{k}-\sum_{i=0}^{k-s}\left(\begin{array}{l}
k \\
i
\end{array}\right)(n-1)^{i},
\end{aligned}
$$

мы видим, что

$$
\begin{aligned}
r g_{s, k-1}-r g_{s-1, k-1} & =\left[n^{k-1}-\sum_{i=0}^{k-s-1}\left(\begin{array}{l}
k \\
i
\end{array}\right)(n-1)^{i}\right]-\left[n^{k-1}-\sum_{i=0}^{k-s}\left(\begin{array}{l}
k \\
i
\end{array}\right)(n-1)^{i}\right] \\
& =\left(\begin{array}{l}
k-1 \\
k-s
\end{array}\right)(n-1)^{k-s} \\
& =\left(\begin{array}{l}
k-1 \\
s-1
\end{array}\right)(n-1)^{k-s} .
\end{aligned}
$$

Отсюда окончательно получаем, что

$$
\operatorname{det}\left(R_{s, k}^{\top} R_{s, k}\right)=n^{\left(\begin{array}{c}
k-1 \\
s-1
\end{array}\right)(n-1)^{k-s}} \operatorname{det}^{n-1}\left(R_{s, k-1}^{\top} R_{s, k-1}\right) \operatorname{det}\left(R_{s-1, k-1}^{\top} R_{s-1, k-1}\right) .
$$

Теорема доказана.

Теорема 3. Справедливо равенство

$$
\operatorname{det} R_{s, k}^{\top} R_{s, k}=n^{s\left(\begin{array}{l}
k \\
s
\end{array}\right)(n-1)^{k-s}} .
$$

Доказательство. По следствию 3,

$$
\operatorname{det} R_{1, k}^{\top} R_{1, k}=n^{k(n-1)^{k-1}} .
$$

Допустим, что формула верна для $(s-1, k-1)$ и $(s, k-1)$, и докажем ее справедливость для $s$ и $k$. Нетрудно видеть, что

$$
\begin{aligned}
\operatorname{det} R_{s, k}^{\top} R_{s, k} & =n^{\left(\begin{array}{c}
k-1 \\
s-1
\end{array}\right)(n-1)^{k-s}} n^{s\left(\begin{array}{c}
k-1 \\
s
\end{array}\right)(n-1)^{k-s}} n^{(s-1)\left(\begin{array}{c}
k-1 \\
s-1
\end{array}\right)(n-1)^{k-s}} \\
& =n^{s\left[\left(\begin{array}{c}
k-1 \\
s-1
\end{array}\right)+\left(\begin{array}{c}
k-1 \\
s
\end{array}\right)\right](n-1)^{k-s}} \\
& =n^{s\left(\begin{array}{c}
k \\
s
\end{array}\right)(n-1)^{k-s} .}
\end{aligned}
$$

Теорема доказана. 
5. Выделим в матрице $T_{s, k}$ базисную систему строк $T_{s, k}^{\mathscr{A}},|\mathscr{A}|=r_{s, k}$, и рассмотрим поведение среднего значения $\alpha_{\mathfrak{A}}\left(T_{s, k}(n)\right)$ квадрата минора матрицы $T_{s, k}^{\mathscr{A}}(n)$.

Базисную систему строк можно представить в виде

$$
T_{s, k}^{A}\left(n_{1}, \ldots, n_{k}\right)=\left[\frac{\mathbf{1}\left(1, n_{1}\right) \otimes T_{(s-1, k-1)}^{A d}\left(n_{2}, \ldots, n_{k}\right)}{E_{n_{1}-1, n_{1}} \otimes T_{(s, k-1)}^{A d}\left(n_{2}, \ldots, n_{k}\right)}\right],
$$

где $E_{n-1, n}-$ единичная матрица порядка $(n-1 \times n)$ с удаленной последней строкой.

Лемма 4. При фиксированном $k u 1 \leqslant s \leqslant k-1 u n \rightarrow \infty$ для средней величины $\alpha_{\mathscr{A}}\left(T_{s, k}(n)\right)$ квадрата минора рангового порядка базисной системы строк матрицы $T_{s, k}$ справедливо равенство

$$
\alpha_{\mathscr{A}}\left(T_{s, k}(n)\right)=(n-1)^{(k-s) / 2}\left[\frac{\left(\begin{array}{c}
k \\
s
\end{array}\right)}{e}\right]^{\left(\begin{array}{c}
k \\
s
\end{array}\right)(n-1)^{k-s}}(1+o(1)) .
$$

Доказательство. Нетрудно видеть, что

$$
\begin{aligned}
\left(\begin{array}{l}
N \\
r
\end{array}\right) & =\left(\begin{array}{c}
n^{k} \\
r
\end{array}\right) \\
& =\frac{1}{\sqrt{2 \pi r}}\left[\frac{n^{k} e}{\left(\begin{array}{l}
k \\
s
\end{array}\right)(n-1)^{k-s}}\right]^{\left(\begin{array}{c}
k \\
s
\end{array}\right)(n-1)^{k-s}}(1+o(1)) .
\end{aligned}
$$

По теореме 3 и формуле (6),

$$
\operatorname{det}\left(T_{s, k}^{\mathscr{A}}(n) T_{s, k}^{\mathscr{A} \top}(n)\right)=n^{s\left(\begin{array}{c}
k \\
s
\end{array}\right)(n-1)^{k-s}} .
$$

Тогда

$$
\begin{aligned}
\alpha_{\mathscr{A}}\left(T_{s, k}(n)\right) & =\operatorname{det}\left(T_{s, k}^{s \mathcal{A}}(n) T_{s, k}^{s \top}(n)\right)\left(\begin{array}{c}
N \\
r
\end{array}\right)^{-1} \\
& =\sqrt{2 \pi r}\left[\frac{\left(\begin{array}{c}
k \\
s
\end{array}\right)}{e}\right]^{\left(\begin{array}{c}
k \\
s
\end{array}\right)(n-1)^{k-s}}\left(\frac{n-1}{n}\right)^{(k-s)\left(\begin{array}{l}
k \\
s
\end{array}\right)(n-1)^{k-s}}(1+o(1)) \\
& =(n-1)^{(k-s) / 2}\left[\frac{\left(\begin{array}{c}
k \\
s
\end{array}\right)}{e}\right]^{\left(\begin{array}{l}
k \\
s
\end{array}\right)(n-1)^{k-s}}(1+o(1)) .
\end{aligned}
$$

Лемма доказана.

Теорема 4 ([14]). Если $1 \leqslant s<k u k \geqslant 3$, то $\alpha_{\mathscr{A}}\left(T_{s, k}(n)\right) \rightarrow \infty$ при $n \rightarrow \infty$ с экспоненичиальной скоростью.

\section{Список литературы}

1. Маркус М., Минк Х., Обзор по теории матриц и матричных неравенств. Наука, Москва, 1972.

2. Воеводин В. В., Кузнецов Ю. А., Матриць и вычисления. Наука, Москва, 1984.

3. Черников С. Н., Линейные неравенства. Наука, Москва, 1968. 
4. Шевченко В. Н., Многогранники многоиндексных транспортных задач: алгебраический подход. В сб.: Материаль конференции «Дискретный анализ и исследование операџий». ИМСО РАН, Новосибирск, 2004, с. 64-70.

5. Шевченко В. Н., Об исследовании миноров матрицы при помощи ее сингулярного многочлена. В сб.: Материалы IX Международного семинара "Дискретная математика и ее приложения» (Касим-Заде О. М., ред.). Изд-во мех.-мат. ф-та МГУ, Москва, 2007, с. 31-34.

6. Шевченко В. Н., Характеристические многочлены многоиндексных транспортных задач. Дискретная математика (2003) 15, №2, 83-88.

7. Емеличев В. А., Ковалев М. М., Кравцов М. К., Многогранники, графы, оптимизация. Наука, Москва, 1981.

8. Титова Е. Б., Шевченко В. Н., Среднее значение квадрата минора матрицы ограничений аксиальной транспортной задачи. Автоматика и телемеханика (2004) №2, 113-117.

9. Титова Е. Б., Шевченко В. Н., Базис правого модуля матрицы ограничений многоиндексной транспортной задачи. В сб.: Материалы конференщии «Математика и кибернетика 2003», Нижний Новгород, 2003, с. 264-265.

10. Титова Е. Б., О среднем значении квадрата базисного минора матрицы ограничений многоиндексной транспортной задачи. В сб.: Тезисы докладов XV международной конференции «Проблемы теоретической кибернетики». Отечество, Казань, 2008, с. 112.

11. Титова Е. Б., Шевченко В. Н., Асимптотика среднего значения квадрата минора матрицы ограничений трехиндексной планарной транспортной задачи. В сб.: Материаль III Всероссийской конференции «Проблемы оптимизачии и экономические приложения». Изд-во ОмГТУ, Омск, 2006, c. 127.

12. Шевченко В. Н., Титова Е. Б., Средняя величина миноров $k$-индексной $s$-валентной транспортной задачи. Информачионный бюллетень Ассочиации математического программирования (2003) 10, 248.

13. Титова Е. Б., Шевченко В. Н., Среднее значение квадрата минора любого порядка матрицы ограничений многоиндексной транспортной задачи. В сб.: Материаль XIV Международной иколь-семинара «Синтез и сложность управляющих систем». Изд-во НГПУ, Нижний Новгород, 2003, с. 81-82.

14. Титова Е. Б., Асимптотика среднего значения квадрата минора базисной подматрицы ограничений многоиндексной транспортной задачи. Информащионный бюллетень Ассощиаџии математического программирования (2007) 11, 215-216.

15. Титова Е. Б., Шевченко В. Н., О минорах матрицы ограничений $k$-индексной $s$-арной транспортной задачи. В сб.: Труды VIII Международной конференции «Дискретные модели в теории управляющих систем». МАКС, Москва, 2009, с. 298-301.

16. Титова Е. Б., Шевченко В. Н., Левые и правые нуль-пространства планарной многоиндексной транспортной задачи. В сб.: Материаль VIII Международного семинара "Дискретная математика и ее приложения». Изд-во мех.-мат. ф-та МГУ, Москва, 2004, с. 229-231.

17. Ильичев А. П., Исследование многогранников многоиндексных транспортных задач, Диссертация на соискание ученой степени кандидата физико-математических наук, Горький, 1988.

Статья поступила 17.02.2010. 